\title{
Are We Ready to Move from Operative to Non-Operative/Preventive Treatment of Dental Caries in Clinical Practice?
}

\author{
N.B. Pitts \\ Dental Health Services Research Unit and Centre for Clinical Innovations, University of Dundee, Dundee, UK
}

\author{
Key Words \\ Clinical management · Dental caries · Treatment \\ planning $\cdot$ Treatment strategies
}

\begin{abstract}
This review focuses on the clinical interactions between patients and the dental team, not on caries prevention at a public health level. Many dentists no longer take a narrow surgical view seeking to apply interventive treatment as a one-off event at a certain trigger point of disease severity and the evidence that caries is an initially reversible, chronic disease with a known multi-factorial aetiology is being appreciated more widely. The caries process should be managed over time in an individualized way for each patient. Very few individuals can be considered to be truly 'caries free' when initial lesions as well as more advanced dentine lesions are considered. It is now very clear that, by itself, restorative treatment of the disease does not 'cure' caries. The caries process needs to be managed, in partnership with patients, over the changing challenges of a lifetime. The answer to the question posed in the title should be, in many cases, that we are ready to move to non-operative/preventive care (if we have not done so already). However, this should be for appropriate stages of lesion extent and in patients who respond to advice on recall frequency and pre-
\end{abstract} ventive behaviours.

Copyright $@ 2004$ S. Karger AG, Basel
The aims of this paper were to bring together the very broad areas of evidence relevant to the important clinical question posed by the organizers and to present this in a framework accessible to an audience including, but not limited to, the caries research community and those undertaking clinical caries management. Although dental public health aspects are mentioned, their full consideration is outside the remit of this review.

In northern Europe, an overtly preventive philosophy has existed for many years. For example, in the Nordic public dental services, recommendations to be circumspect with operative intervention have long been in place. Although the overall decline in caries can be attributed to a range of factors outside the impact of direct dental care, not least the widespread use of fluoride toothpastes, a more restrained approach to placing the first restoration has also been seen to have a beneficial impact on oral health [Heidmann et al., 1987]. In the USA, however, Ismail et al. [2001] found that much of general dentistry is still within the 'restorative era', although there is a growing interest in preventive management.

Since 1998, pilot studies of new forms of general dental practice service - the personal dental services - have been made in England [MacLeod et al., 2003]. Some of the studies provided an opportunity for the personal dental services dentists to promote a less interventionist approach to dental care. One study reduced the rate of filled teeth (from 81 to 61 per 100 adult registrations) and then

Prof. N.B. Pitts

Dental Health Services Research Unit, The Mackenzie Building

Kirsty Semple Way

Dundee DD2 4BF (UK)

Tel. +44 1382 420067, Fax +44 1382 420051, E-Mail n.b.pitts@dundee.ac.uk 


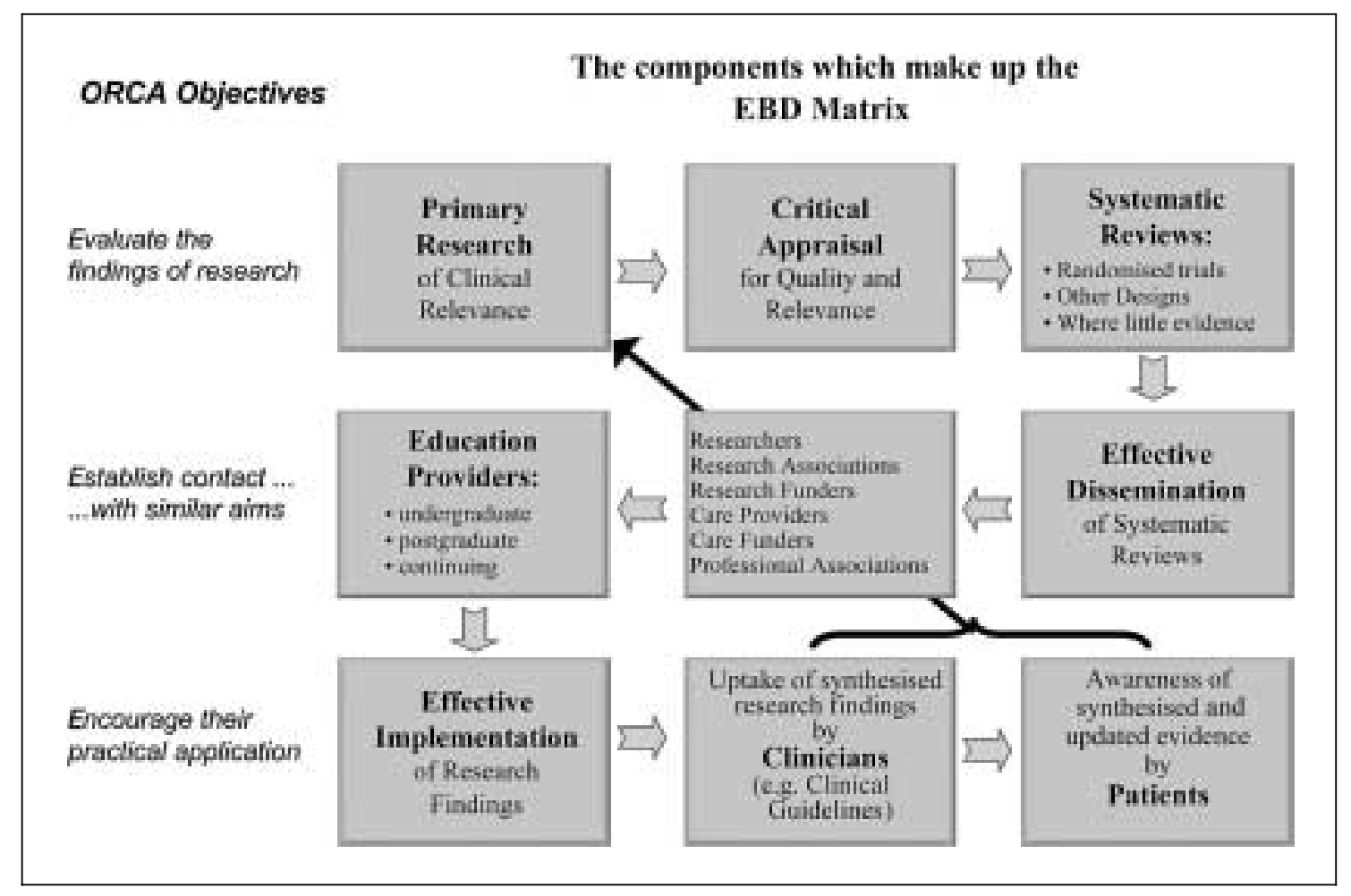

Fig. 1. The evidence-based dentistry (EBD) matrix and ORCA's objectives.

sustained the low rate compared with local general dental practitioners. The capitation-based studies illustrated the potential for dentists to change their prescribing behaviour and provide care which was commonly viewed as being in the long-term benefit of their patients [MacLeod et al., 2003].

If we are to ascertain how to manage caries, we must, in this era of evidence-based health care (EBH) and evidence-based dentistry (EBD), reflect both systematic reviews (SRs) of the literature (the objective state of the science) as well as expert and consensus views on clinical practice (the state of the art). The science and the art can then be integrated into a rational framework for clinical practice, which will determine the pace of the move from operative to non-operative/preventive treatment.

The move to EBH is a global phenomenon, but is taking place at a variety of speeds in different countries. The EBH philosophy requires a more open approach from health professionals. Higher value is now given to robust research findings. Lower quality research and expert opinion alone are given limited credence but become important guides in areas where there is no high quality research relating directly to the clinical question. In a number of countries, patients and their views are also becoming increasingly empowered, for example in the Options for Change developments in England [Department of Health, 2002]. Research in a particular field is now objectively assessed and synthesised by formal SRs, ideally of randomised clinical trials. EBD is designed to help the clinician and patient when addressing specific clinical dental questions. It has been usefully defined by the American Dental Association [American Dental Association, 2002] as 'an approach to oral health care that requires the judicious integration of (1) systematic assessments of clinically relevant scientific evidence, relating to the patient's oral and medical condition and history, with (and this is the part that is often missed) (2) the dentist's clinical expertise and (3) the patient's treatment needs and preferences.'

To fulfil these requirements, a body of knowledge must first be identified, then objectively synthesised, before being communicated to clinicians and their patients in ways that encourage effective implementation of evidence in practice. Figure 1 is a flow chart illustrating the various 
processes which together make up EBD. It also shows how the resulting clinical practice should then feed back to new primary research evaluating the new practice once again. This figure also demonstrates that the modern EBD approach to oral health care is in fact very well served by the formal objectives of ORCA.

\section{Scientific Background - Summary}

In order to review, discuss and advance clinical caries management, it is imperative to agree on and employ modern definitions of the disease process and diagnosis of the disease which are both scientifically and clinically viable. This area was considered at length at the International Consensus Workshop on Caries Clinical Trials [Pitts and Stamm, in press]. The agreed consensus statements relating to disease definition express that: (1) the caries process occurs as an interaction between the biofilm and the tooth surface and subsurface; the caries lesion is the manifestation of the stage of the process at one point in time; (2) caries progression occurs when the demineralisation and remineralisation equilibrium is out of balance, leading to net mineral loss, and (3) remineralisation can arrest or reverse progression of disease and can lead to changes in mineral quality; the understanding of the caries process has progressed far beyond the point of restricting the evidence for dental caries to the $\mathrm{D}_{2}$ (caries in enamel only) or $\mathrm{D}_{3}$ (caries in enamel and dentine) levels of cavitation.

The agreed consensus statements relating to the detection and diagnosis of caries express that there is considerable confusion with the terminology employed in the literature. The three agreed key terms of direct relevance to preventive caries care were: (1) lesion detection: implies an objective method of determining whether or not disease is present; (2) lesion assessment: aims to characterise or monitor a lesion, once it has been detected, and (3) caries diagnosis: should imply a human, professional, summation of all available data.

It is now appreciated that caries is an initially reversible, chronic, disease process with a known multi-factorial aetiology [Fejerskov and Manji, 1990; Kidd, 1996; Baelum and Fejerskov, 2003]. The stages of the caries process, including clinical lesions in enamel, are well documented over many years [Backer-Dirks et al., 1951; Marthaler, 1965; Møller, 1966; Silverstone, 1973; World Health Organization, 1979; Manji et al., 1991; Kidd, 1996; Fejerskov et al., 2003].
The state of the art in understanding lesion detection recognises that very few individuals can be considered to be truly 'caries free'. This is not a new observation; Magitot [1886] some 118 years ago suggested that we switch our focus away from defining dental caries only as 'cavities' and should recognise the value of detecting the stages of the caries process.

The stages of caries have been represented graphically as an iceberg of dental caries experience [Pitts, 1997a], which is summarised in a simplified form as figure 2 . The earliest changes to the dental enamel (at the base of the iceberg) are subclinical, subsurface demineralisations [Fejerskov et al., 2003], often at inaccessible sites. These lesions are extremely common and can often be found when apparently sound surfaces on extracted teeth are subsequently examined histologically. The next level (or diagnostic threshold) up comprises lesions which can be visualised as caries in enamel, usually with apparently 'intact' surfaces. Because of the intrinsic insensitivity of unaided visual detection, a proportion of these lesions will be unseen by even a diligent examiner, and thus 'missed'. There is clear evidence that these non-cavitated enamel lesions are a 'stage' of dental caries and not some predisease state [Kidd, 1996]. A smaller number of surfaces in the same individual may have discernible lesions in dentine; again a proportion of these will be unseen and therefore missed during clinical examination. Finally, at the tip of the iceberg are the comparatively extensive and more clinically obvious lesions extending into the dental pulp.

Although many values for caries prevalence and incidence are expressed using the DMF index, the values reported will not be directly comparable if different diagnostic thresholds have been used. Diagnostic thresholds vary significantly between different types of research, as well as between traditional epidemiological surveys and clinical practice [Murray and Pitts, 1997]. Although this trap has been recognized within caries research circles for many years [Backer-Dirks et al., 1951; Moller, 1966; Pitts and Fyffe, 1988], it is only recently that clinicians, dental public health workers, service planners and patients are starting to appreciate these complexities with debate around the outcomes of preventive caries control strategies, which seek to prevent the need for fillings wherever possible. Thus, figure 2 demonstrates how the 'dentineonly $\mathrm{D}_{3} \mathrm{MF}$ ', used traditionally as an epidemiological measure of caries prevalence, will inevitably produce a lower value than 'enamel + dentine $\mathrm{D}_{1} \mathrm{MF}$ '. The latter in turn will produce lower values than 'enamel + dentine $\mathrm{D}_{1} \mathrm{MF}$ ' used together with diagnostic aids, such as bite- 


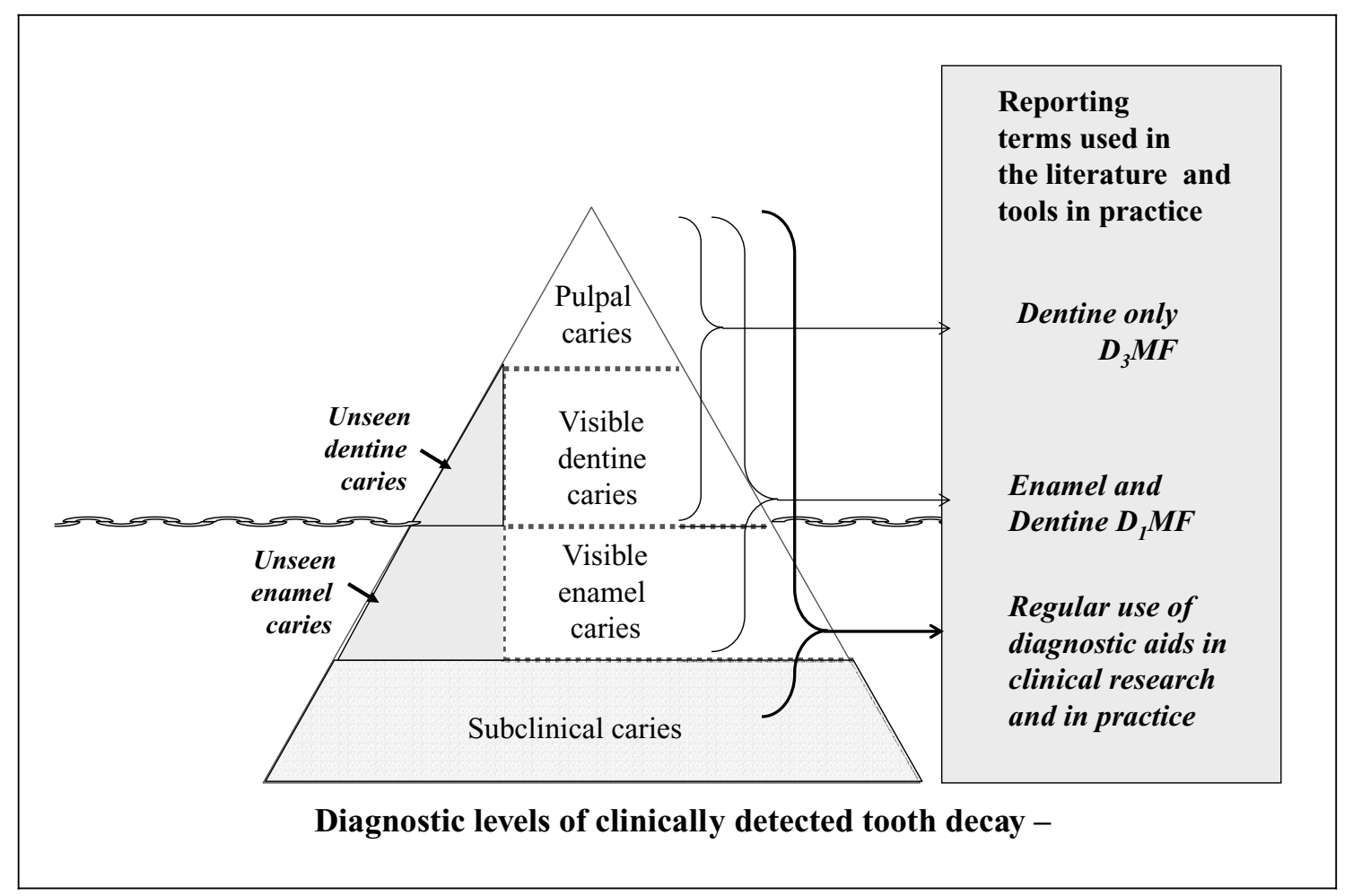

Fig. 2. Diagnostic levels of clinically detected tooth decay - use in the literature and in clinical practice using visual methods of caries detection.

wing radiographs [Murray and Pitts, 1997; Machiulskiene et al., 1999].

The preventive methods available to the dental team range from topically applied fluorides in a variety of forms (with toothpastes and varnishes being found to be particularly effective, while rinses and tablets are becoming less favoured); a focus on helping the patient achieve optimal plaque control (particularly on occlusal surfaces during eruption); strategies to modify diet with respect to the frequency of sugar consumption, and methods of increasing the resistance of the tooth, such as pit and fissure sealants [National Institutes of Health, 2001].

\section{Existing Reviews and Consensuses}

SRs of randomised clinical trials (RCTs) judged to be of high quality and comparing operative with non-operative intervention for caries directly are unfortunately very sparse. However, there are a number of more general SRs and a range of differing types of evidence evaluating the individual elements which comprise operative and non- operative care. As with many SRs in medicine, the quality of studies reported is very variable and there are more studies which are of poor quality by modern standards than is desirable. There are comparatively few data on the patient or quality-of-life aspects of these two approaches to clinical caries management.

The Cochrane Oral Health Group, part of the International Cochrane Collaboration, undertakes and maintains an ever-widening series of robust systematic reviews on RCTs of interventions in oral health [The Cochrane Library, 2003]. The attention to methodological detail and the international refereeing process ensures that for RCTs these reviews are seen as the gold standard.

A step forward in documenting and assessing the evidence in the area of the question posed here was the National Institutes of Health (NIH) Consensus Development Conference on Diagnosis and Management of Dental Caries throughout Life [National Institutes of Health, 2001; http://www.consensus.nih.gov], which went to great lengths to control bias and adopted rigorous processes for SRs. The Journal of Dental Education published a whole issue devoted to the Consensus Development Conference 
[National Institutes of Health, 2001], which included a full SR commissioned by NIH from the Research Triangle Institute [Bader et al., 2001], as well as 31 other SRs and semi-systematic reviews together with invited external expert comments on the Research Triangle Institute review. Although some aspects considered by the NIH were broader than the review topic here and new findings have been reported since March 2001, the Consensus Development Conference report still gives an excellent overview of clinical caries research.

Other relevant SRs are those from the International Consensus Workshop on Caries Clinical Trials [Pitts and Stamm, in press], the Scottish Intercollegiate Guidelines Network [Scottish Intercollegiate Guidelines Network, 2000], the National Institute for Clinical Excellence [National Institute for Clinical Excellence, in press], and the Swedish Council on Technology Assessment in Health Care [http://www.sbu.se].

\section{State of the Art}

The question posed about how best to manage caries refers to selecting an appropriate treatment (or care) philosophy. In order to plan appropriate clinical caries management, a firm foundation of knowledge about caries detection, assessment and then diagnosis is required. As the following sections will discuss, clinicians should ideally unite: (a) a thorough and up-to-date understanding of evidence about the pathogenesis of caries [Fejerskov et al., 2003]; (b) an understanding of when to use appropriate diagnostic levels [Kidd et al., 2003], and (c) an ability to interpret evidence and clinical information derived from all sources [Pitts, 1997; Kidd and Nyvad, 2003; National Institute for Clinical Excellence, in press]. While this synthesis of related information and evidence seems straightforward and uncontentious to some, it is viewed as radical and somewhat alien by others. This is not surprising when the variations in the teaching content in this area of undergraduate and continuing education across countries and health systems are considered. A further threat to understanding in this clinical field is a failure to be clear about the focus of a clinical or research question. These can be posed from a surface or tooth perspective, from the individual patient perspective or from the population, community or society perspective [Baelum and Fejerskov, 2003]. All are appropriate in certain situations.

In the restorative era, the decision process for managing caries centred on an almost unconscious planning of which surface to fill with what material [Bader and Shugars, 1995; Ismail et al., 2001], and this approach remains in use with many in general dental practice today. However, many dentists in many geographic locations are no longer taking only a narrow surgical view, seeking to apply interventive treatment as a one-off event at a certain trigger point of disease severity. Classical restorative treatment of the disease only surgically removes the carious tissue and does not, by itself, result in a 'cure' for caries. There is established evidence that, although the time frames vary, once a restoration is placed [NHS Centre for Reviews and Dissemination, 1999], the tooth is likely to be subjected to a series of replacement restorations, tending to increase in size, complexity and cost. The limitations of 'repeat restorative alone' care [Elderton, 1990; Mjör and Toffeneti, 2000] are increasingly acknowledged and replicated worldwide. Restorations have been shown to fail by a number of factors, with inadequate preparation, marginal failure of the restoration or tooth and secondary caries at approximal sites being the principal problems.

It is clear that, at the individual patient level, the disease process of dental caries has to be managed over an extended time by combating each of the multiple known aetiological factors [Kidd and Nyvad, 2003]. It should be appreciated that this discussion about how best to manage and control the disease of dental caries at the encounter between patient and dental health professional in no way undercuts the importance of broader health and community interventions for primary prevention at the population level 'upstream' from the dentist. These interventions, combined with oral health promotion linked to other non-dental health promotion activities should (if these are delivered effectively) ensure that the burden of disease for the population (and thus those attending dental clinics and surgeries) is reduced. The public health aspects are beyond the scope of this review but need not be at odds with clinically effective preventive dental care delivered in a primary care setting.

From the key SRs of caries prevention [The Cochrane Library, 2003] and management (such as those presented at the NIH Consensus Development Conference [National Institutes of Health, 2001]), we know that in addition to the improvements in caries associated with water fluoridation, fluoride toothpaste and public health initiatives, there have been successes at the practice level in reducing caries prevalence and that 'effective professional practices such as the use of fluoride, sugarless products and dental sealants were reconfirmed' [National Institutes of Health, 2001]. However, the Consensus Development Confer- 
ence also warned of inadequacies in current diagnostic practices: limitations which have to be borne in mind when making clinical decisions. The consensus statement's section on clinical decision making was that 'current information indicated that the opportunity now exists to extend prevention and treatment of caries to nonsurgical methods. These include prevention, remineralisation, and arrest of early non-cavitated lesions' [National Institutes of Health, 2001]. The panel went on to point up the need for reimbursement methods to reward non-surgical treatment and for educational institutions and accreditation bodies to support the growing evidence for prevention and non-surgical treatment, where indicated.

The state of the art in modern clinical disease management is to use evidence-based clinical guidelines to inform the dentists and patients when they need to have an up-to-date synthesis of recent research findings [National Institutes of Health, 2001]. The Scottish Intercollegiate Guidelines Network [Scottish Intercollegiate Guidelines Network, 2001; www.sign.ac.uk] has been producing such guidelines to aid medical practice since 1993 and is an example of this approach to facilitating evidence-based care. In 2000, they published a guideline on providing appropriate care for 6- to 16-year-old children attending dental practices in Scotland [Scottish Intercollegiate Guidelines Network Guideline, 2000]. This recommends a preventive approach to clinical management. One of its key recommendations is that, because of the 'polarisation' of the population into a low-caries majority and a cariesactive minority, 'an explicit caries risk assessment should be made for each child presenting for dental care'.

The polarisation phenomenon [Poulsen et al., 2001] as caries declines is important. Changes in Danish 15-yearold children between 1980 and 1995 show that: "with increasing divergence from a Poisson distribution, caries risk is itself skewed. This means that high risk groups would continue to have high risk to future caries attack and progression' [Poulsen et al., 2001]. This does not mean that those at the lower-caries end of the distribution are not at risk of new disease or caries progression. This is why it is important to have both a public health strategy to continue to pull the distribution even further to the low-caries side for everyone [Sheiham and Fejerskov, 2003] - and an element of high-risk strategy to provide appropriate care for those with a high burden of disease. When patients present at dentists for clinical care, it is important that risk is considered so that care is tailored to the needs of the individual [Kidd and Nyvad, 2003]. The following factors should be considered when assessing caries risk: medical history; social history, especially socioeconomic status; clinical evidence of previous disease; dietary habits, especially frequency of sugary food and drink consumption; use of fluoride; plaque control, and salivary factors [Scottish Intercollegiate Guidelines Network Guideline, 2000; Kidd and Nyvad, 2003].

Although there has been a focus on children in defining this type of preventive care, and the bulk of the available evidence comes from studies of children and adolescents, it should be appreciated that both adults and children are important and that at all ages the disease is driven predominantly by the intensity of the cariogenic challenge for the individual at a single time rather than by chronological age alone.

The philosophy of 'wellness management' is already the state of the art in some communities and is increasingly prominent in US health care. This reflects both an attempt to ensure that preventive care is optimised by early intervention and an understanding of many patients' increasing desire to optimise and maintain health, rather than to suffer from a disease and its consequences. This philosophy is gaining support amongst consumer groups and some of those seeking to respond to spiralling general health care costs. Under this model of care, waiting until you have a disease is too late; one needs to pro-actively modify risk factors to avoid the transitions to the diseased state, or to catch the disease at an earlier stage in its development. This approach may have dental applications, but clinical outcome studies are needed.

The model of care underlying the clinical management of dental caries is changing from the traditional, but increasingly outmoded one in which the dentist restoring caries is seen as a surgeon, to the model which sees the dentist as a physician. The physician and team are concerned with the prevention and control of the disease wherever possible, as opposed to the surgeon, who manages caries by the restoration and re-restoration of teeth.

Traditionally the dentist as a surgeon provided treatment based upon excision of diseased tissue, detection/ intervention, treating signs and symptoms, rehabilitation of lost tissue with early intervention and extension. By contrast, the dentist as a physician now provides care based on: health maintenance; early detection and monitoring; controlling causal agents; use of appropriate pharmacological agents, and minimal intervention. These two roles are, however, not mutually exclusive as, depending on the needs of the patient and the outcome of professional and self-care, the dentist may have to fulfil both roles for the same patient. Although the term 'medical model of caries care' has been used widely in this more preventive 
Fig. 3. The iceberg of dental caries linked to contemporary patient advice and treatment need [Pitts and Longbottom, 1995].

\section{The 'iceberg of dental caries' (1995>)}

by contemporary patient advice and treatment need

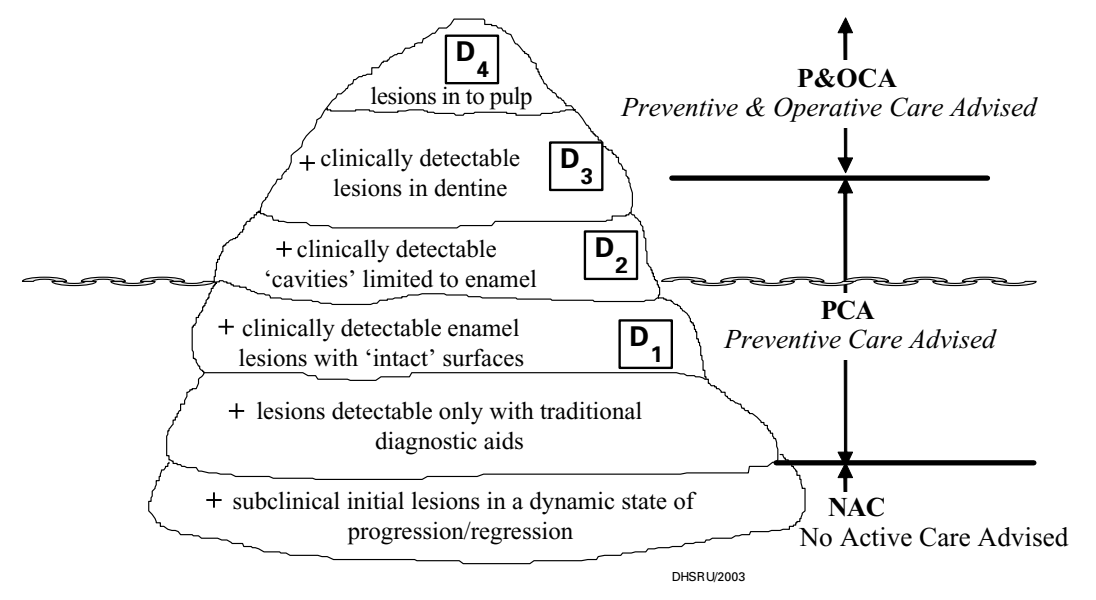

clinical caries management context [National Institutes of Health, 2001], this has been taken to imply an almost exclusive focus upon caries as an infectious disease and, in turn, an antimicrobial approach. As the caries process has a multi-factorial aetiology [Fejerskov and Manji, 1990], it is preferable to use a broader approach (the dentist/team as physician model), which allows a clearer role for oral hygiene and diet-related preventive measures, as well as antibacterial and pharmacological interventions in caries control.

A care philosophy which integrates all the scientific information underpinning modern clinical caries management is summarised in figure 3 , which, like figure 2, illustrates the iceberg phenomenon, but this time linked to contemporary patient advice and treatment need [Pitts and Longbottom, 1995]. The two more extreme care treatment options are the most straightforward. Very small subclinical initial lesions require no active care in many lower-caries risk patients who need only surveillance at appropriate, individualised time intervals. For more caries-active individuals, the appearance and progression of small lesions are a marker of disease activity and an indication that more aggressive preventive care is required urgently. At the other end of the spectrum, the presence of large $\mathrm{D}_{4}$ lesions extending into the pulp indicates that some form of operative intervention or care is required urgently and this should be advised in addition to the preventive care advice.
Determining the cut-offs at both ends of the severity scale for the intermediate group of lesions, which require only preventive care advised, is more complex and contentious. These cut-offs have to be decided for individual lesions and patients by dentists utilising all the information available from the diagnostic assessments, the patient and the medical, dental and social histories. Clinical preventive care is focussed on ensuring, at an early stage, that new disease can be avoided if possible, and limited or controlled if it cannot be. The potential long-term wellbeing, health and economic benefits for individual patients are immense.

One aspect highlighted by this overview is the urgent need in many countries for improving education about cariology. There are concerns over the lack of basic science-led theory and preventive practice in clinical cariology when compared to other disciplines taught in undergraduate schools, as well as gaps in postgraduate and continuing dental education [Ismail et al., 2001]. This often produces widespread and alarming ignorance among graduates about both the basic science of the disease process and the clinical strategies to best manage the disease. Education of the wider dental profession and the public, patients and planners on the potential for caries prevention, is also poor in many countries. Examples include the continuing misuse and misunderstanding of terms such as 'caries-free', a lack of appreciation of the continuing risk of caries throughout life and a failure to 
understand that the minority of those who still have dental disease carry very significant quality-of-life burdens.

The key point for clinical caries management is that the caries process needs to be managed for each individual by the dental team, in partnership with the patient, over the changing and continuing cariogenic challenges of a lifetime. The frequency of visits at which assessments should be made should reflect the particular needs of the individual patient taking into account current caries status and an individually determined prognosis [Kidd and Nyvad, 2003; National Institute for Clinical Excellence, in press].

\section{Future Research}

We need to increase our scientific understanding of the caries process so that it can be prevented and controlled more effectively. For this reason, we need rigorous research to better understand caries aetiology, pathogenesis, activity, detection (informing diagnosis), diagnosis, monitoring (informing prognosis with minimal harm) and risk assessment [National Institutes of Health, 2001]. This research will then inform the development of new adjuncts to preventive caries control, which will need to be tested in realistic clinical settings.

Key recommendations of the 2001 NIH Consensus Development Conference for future research were: (1) epidemiology of primary and secondary caries to collect information on natural history, treatment and outcomes across ages; (2) clinical trials, to modern standards, of established and new treatment methods; (3) studies of clinical practice with respect to effectiveness, appropriateness, quality of care, outcomes and health-related quality of life, and (4) studies to identify genes and genetic markers of diagnostic, prognostic and therapeutic value.

There have been enormous changes in design and statistical analysis of medical clinical trials, as well as in the ethical, economic and regulatory environments over the last 20 years. Despite the dramatic changes in our knowledge about research, epidemiology, detection systems and the effectiveness of clinical care for dental caries over the same period, there have been no coordinated formal initiatives to review the methodological requirements for RCTs of caries-preventive interventions [Pitts and Stamm, in press]. One consensus statement expressed the view that recording only cavitated lesions as an outcome measure is becoming outmoded, and that for future caries clinical trials, recording of non-cavitated lesions is essential [Pitts and Stamm, in press].

Moving from Operative to Preventive

Clinical Caries Management?
To specifically advance non-operative clinical caries management, we need research into the effectiveness of preventive interventions and caries control for individuals [National Institutes of Health, 2001]. This should bring together basic science and applied technology.

A key area of clinical need where more research is required is on lesion assessment, which ascertains the 'activity' of caries in individuals and at specific sites [Pitts and Renson, 1987]. At present, most research in this area is concerned with assessing changes in lesion characteristics over specific time periods, but this could and should be complemented with valid assessments of whether or not a lesion is active, made on a single occasion. This has recently been attempted clinically by Nyvad et al. [1999, 2003]. What is needed is a way of differentiating active from inactive lesions and it is important to highlight the different types of behaviour that may be exhibited. The clinical decisions that would be made would clearly be different between the active and inactive lesions if this information were known. Similarly, the optimal timing of care and the planning of individualized recall intervals would be enhanced by this type of information. It would also be very useful for both clinical practice and research studies to be able to reliably identify each type of active and inactive lesion.

One of the successes of ORCA is the range of international collaborations that have been initiated and developed between members from a range of different countries. This tradition will remain important in order to achieve generalisable and valid research findings. A recent initiative is the formation of a committee which has started the development of an international caries detection and assessment system [Pitts, in press]. The idea is to work, to develop and test an open framework, which will allow sufficient standardisation of caries criteria to facilitate future SRs. The system will provide a range of methodological tools based on a synthesis of the published literature and suitable for a variety of applications. The aim is to bring together methodology from epidemiological surveys, clinical research and clinical practice. These unifying, predominantly visual criteria would code the characteristics of clean, dry teeth. They will be able to record both enamel and dentine caries and, in future research, should help to explore the measurement of caries activity. It is hoped that the development and use of the international caries detection and assessment system might lead to better quality information to inform decisions about appropriate diagnosis, prognosis and clinical management at both the individual and ultimately public health levels. It should also provide a common framework to val- 
idate and explore the impact of a number of detection aids such as explorers, magnification, tooth separation, fibre optic transillumination, radiography and other diagnostic aids. New studies in these areas are still needed as the synthesis of the results of many existing studies is compromised by the conflicting criteria and methodologies employed [Bader et al., 2001]. A further problem to be overcome is the validation of caries registration methods [Sjogren et al., 2003].

In the future, there should also be more research into treatment choices for clinical caries management. These should include studies of the dynamics of communications and behaviour change, in both patients and health professionals. Such studies should be linked with outcome evaluations to see if the potential of health informatics and clinical decision support [Department of Health, 2002] can be realised. Assessing cost effectiveness as well as clinical effectiveness will be important in determining how well preventive caries interventions can compete for scarce health resources.

In routine dental care, choices are frequently made between alternative approaches. The choices between preventive and restorative alternatives are not always easy or straightforward and can be important [National Institutes of Health, 2001; Department of Health, 2002]. The financial arrangements for the dentist, dental team and patients in caries management have often evolved from historical tradition in each country. Most systems have not explicitly asked what is the evidence supporting each option and how it matches the financial incentives for the dentists, the dental team and the patients receiving care. Research is needed to try to inform dental public planning, which should seek to ensure that dental remuneration systems and incentives are linked to optimal and effective caries prevention and control.

Recently, the English Department of Health [2002] published a radical document and subsequent legislation is bringing about changes in the organisation of dental services, dentist remuneration and the way in which oral health is assessed. A key aim of this modernisation process is to move towards a more preventively oriented and clinically effective way of meeting patients' needs [Pitts, 2003]. Under the new arrangements, a comprehensive oral health assessment will comprise three elements: diagnosis, prevention and initial treatment planning. The changes to the remuneration system are designed to 'remove existing perverse incentives' which favour restorative care.

Appropriate strategies for use in developing countries are also required so that the understandable desire to have equity with and copy the 'developed' countries does not result in the introduction of inappropriate and inefficient types of caries prevention and control. The restoratively focused era of the 1960s to the 1980s in the developed world is not a desirable model to copy, as caries prevalence and incidence have now changed, as has our knowledge about dental caries and its control [National Institutes of Health, 2001]. Cost-effective and locally appropriate preventive approaches should be adopted from the outset and should meet local objectives.

\section{Potential Impact on Clinical Practice}

Non-operative caries management supported by meticulous caries detection, lesion assessment and diagnosis, when combined with modern, proven, caries prevention technologies, should change the face of routine clinical practice. Dentists can and should help their patients control caries preventively and manage their oral health [Kidd and Nyvad, 2003].

Primary caries prevention (preventing new disease) will be an important activity at the population, group and individual levels. For patients attending a dentist, secondary caries prevention - the early detection and prompt, efficacious treatment of disease - is likely to be improved in the coming years by the introduction of new technologies. In more and more patients, this type of clinical care should prevent many lesions from ever reaching the stage when operative intervention is needed [Fejerskov and Nyvad, 2003]. From the patients' perspective, the realisation that the need for many fillings can be avoided entirely will be very attractive.

When tertiary prevention and operative intervention are clearly needed, careful instrumentation maintaining more sound tooth structure, combined with the introduction of 'smart' materials with better physical properties, should improve our very limited ability at durable repair [Ismail et al., 2001]. The incorporation of cariostatic materials (such as slow-release fluorides) within restorations has the potential to aid continuing caries control in the future, provided more sustained low-dose fluoride delivery can be achieved in vivo. The use of slow-release fluoride reservoirs bonded to tooth structure is also an attractive prospect for changing clinical practice.

EBD should combine with new knowledge services and information technology to ensure that quality-assured objective information about prevention, disease control and treatment choices are readily available and accessible to both patients and the dental team. The gap between research and practice should be reduced in time. 
Non-operative caries care should be more clinically effective, appropriate to patients' needs and more costeffective than traditional operative care. This new type of care should be provided within a number of new delivery systems in several countries as the design and economics of health care delivery systems and insurance contracts evolve. Funding systems will change, and are already doing so, in response to changes in disease patterns, the evidence base and professional and patient expectations [Department of Health, 2002].

The recommendations made in this non-systematic review must be graded at the consensus or expert opinion level. This is below that which would be accorded to specific meta-analyses of high-quality SRs focusing only on this question, but we do not yet have such reviews.

Taking into account the evidence reviewed above, we are, in many cases, ready to move to non-operative/preventive caries treatment in clinical practice if we have not done so already. This style of care should be optimal for appropriate stages of lesion extent when surfaces are macroscopically intact, and with co-operative patients. Where significant cavity formation and disease progression are seen, operative intervention is still indicated. This should, however, be minimally invasive and executed to a high technical standard. In the future, it should be technically possible, through partnerships between patients, the dental profession, society and industry, for a patient of any age to benefit from high-quality, on-going, preventive caries care in clinical practice. The clinical team should be up to date with the developing evidence. Treatment should be based on a thorough diagnostic work-up, which would help dentists and patients agree to individualised, preventive, caries control plans to manage and control the disease process throughout life.

\section{Acknowledgements and Disclaimer}

Thanks are due to Dr. Chris Longbottom, with whom the PCA/ OCA caries management approach has been developed. The views expressed are those of the presenter and do not necessarily reflect those of any UK Health Department, the Medical Research Council or any partner or sponsor of the Centre for Clinical Innovations.

\section{References}

American Dental Association Policy on EvidenceBased Dentistry: American Dental Association Position Statements, 2002:www.ada.org/prof/ resources/positions/statements/evidencebased.asp.

Backer-Dirks O, van Amerongen J, Winkler KE: A reproducible method for caries evaluation. $\mathrm{J}$ Dent Res 1951;30:346-359.

Bader JD, Shugars DA: Variation in dentists' clinical decisions. J Public Health Dent 1995;55: 181-188.

Bader JD, Shugars DA, Bonito AJ: Systematic reviews of selected dental caries diagnosis and management methods. J Dent Educ 2001;65: 960-968.

Baelum V, Fejerskov O: Caries diagnosis: A mental resting place on the way to intervention?; in Fejerskov O, Kidd EAM (eds): Dental Caries The Disease and Its Clinical Management. London, Blackwell Munksgaard, 2003, pp 101110.

Department of Health: NHS Dentistry: Options for Change. London, Department of Health, 2002. www.doh.gov.uk/cdo/optionsforchange.

Elderton RJ: Clinical studies concerning re-restoration of teeth. Adv Dent Res 1990;4:4-9.

Fejerskov O, Manji F: Risk assessment in dental caries; in Bader JD (ed): Risk Assessment in Dentistry. Chapel Hill, University of North Carolina Dental Ecology, 1990, pp 215-217.
Fejerskov O, Nyvad B: Is Dental Caries an Infectious Disease? Diagnostic and Treatment Consequences for the Practitioner. Nordic Dentistry 2003. Copenhagen, Quintessence Copenhagen, 2003, pp 141-152.

Fejerskov O, Nyvad B, Kidd EAM: Clinical and histological manifestations of dental caries; in Fejerskov O and Kidd EAM (eds): Dental Caries - The Disease and Its Clinical Management. London, Blackwell Munksgaard, 2003, pp 7197.

Heidman J, Holund U, Poulsen S: Changing criteria for restorative treatment of approximal caries over a 10-year period. Caries Res 1987;21: 460-463.

Ismail AI, Hasson H, Sohn W: Dental caries in the second millenium. J Dent Educ 2001;65:953959.

Kidd EAM: The carious lesion in enamel; in Murray JJ (ed): Prevention of Oral Disease, ed $3 .$. Oxford, Oxford University Press, 1996, pp 95106.

Kidd EAM, Nyvad B: Caries control for the individual patient; in Fejerskov O, Kidd EAM (eds): Dental Caries - The Disease and Its Clinical Management. London, Blackwell Munksgaard, 2003, pp 303-312.

Machiulskiene V, Nyvad B, Baelum V: A comparison of clinical and radiographic caries diagnoses in posterior teeth of 12-year-old Lithuanian children. Caries Res 1999;33:340-348.
MacLeod HST, Morris AJ, Hill KB: Evaluation of personal dental services (PDS) first wave pilots: The alternative to general dental services (GDS) offered by the capitation based pilots. Br Dent J 2003;195:644-650.

Magitot E: Therapeutic indications in dental caries. Part 1. Br J Dent Sci 1886;29:405-410.

Manji F, Fejerskov O, Baelum V, Luan W-M, Chen $\mathrm{X}$ : The epidemiological features of dental caries in African and Chinese populations: Implications for risk assessment; in Johnson NW (ed): Risk Markers for Oral Diseases. Dental Caries Markers of High and Low Risk Groups and Individuals. Cambridge, Cambridge University Press, 1991, vol 1, pp 62-100.

Marthaler TM: The caries-inhibiting effect of amine fluoride dentifrices in children during three years of unsupervised use. Br Dent $\mathrm{J}$ 1965;119:153-163.

Mjör I, Toffenetti F: Secondary caries: A literature review with case reports. Quintessence Int 2000;31:169-71.

Møller IJ: Clinical criteria for the diagnosis of the incipient carious lesion. Adv Fluorine Res 1966;4:67-72.

Murray JJ, Pitts NB: Trends in oral health; in Pine CM (ed): Community Oral Health. Dundee, University of Dundee, Reed Educational and Professional, 1997, pp 126-146. 
National Institutes of Health: The diagnosis and management of dental caries throughout life. National Institutes of Health Consensus Development Conference, Washington March 26th28th 2001. J Dent Educ 2001;65:1162-1168.

National Institute for Clinical Excellence: Dental Recall: Recall Interval between Routine Dental Examinations - Guideline. London, National Institute for Clinical Excellence (NICE), Department of Health, in press.

NHS Centre for Reviews and Dissemination: Restoration Longevity: Effectiveness Matters Bulletin. York, 1999.

Nyvad B, Machiulskiene V, Baelum V: Reliability of a new caries diagnostic system differentiating between active and inactive caries lesions. Caries Res 1999;33:252-260.

Nyvad B, Machiulskiene V, Baelum V: Construct and predictive validity of clinical caries diagnostic criteria assessing lesion activity. J Dent Res 2003;82:117-122.

Pitts NB: Review of ICW-CCT meeting and philosophy and approach of ICDAS. Proc Third Indiana Conf Early Detect Dent Caries, Indiana, in press.
Pitts NB, Fejerskov O, von der Fehr FR: Caries epidemiology, with special emphasis on diagnostic standards; in Fejerskov O, Kidd EAM (eds): Dental Caries - The Disease and Its Clinical Management. London, Blackwell Munksgaard, 2003, pp 140-163.

Pitts NB, Fyffe HE: The effect of varying diagnostic thresholds upon clinical caries data for a low prevalence group. J Dent Res 1988;67:592596.

Pitts NB, Longbottom C: Preventive Care Advised (PCA)/Operative Care Advised (OCA) - Categorising caries by the management option. Community Dent Oral Epidemiol 1995;23:5559.

Pitts NB, Renson CE: Monitoring the behaviour of posterior approximal carious lesions by image analysis of serial standardised bitewing radiographs. Br Dent J 1987;162:15-21.

Pitts NB, Stamm JW: Proceedings of the International Consensus Workshop on Caries Clinical Trials ICW-CCT. J Dent Res, in press.

Poulsen S, Heidman J, Vaeth M: Lorenz curves and their use in describing the distribution of 'the total burden' of dental caries in a population. Community Dent Health 2001;18:68-71.

Scottish Intercollegiate Guidelines Network: SIGN guidelines: A Guideline Developer's handbook. Edinburgh, Scottish Intercollegiate Guidelines Network, 2001, SIGN publication No 50.
Scottish Intercollegiate Guidelines Network Guideline: Targeted Caries Prevention in 6- to 16Year-Olds Attending for Dental Care. Edinburgh, Scottish Intercollegiate Guideline Network, 2000.

Sheiham A, Fejerskov O: Caries control for populations; in Fejerskov O, Kidd EAM (eds): Dental Caries - The Disease and Its Clinical Management. London, Blackwell Munksgaard, 2003, pp 313-325.

Silverstone LM: Structure of carious enamel including the early lesion. Oral Sci Rev 1973;3: 100-160.

Sjogren P, Ordell S, Halling A: Validation methodology in publication describing epidemiological registration methods of dental caries: A systematic review. Community Dent Health 2003;20: 251-259.

Swedish Council on Technology Assessment in Health Care: http://www.sbu.se.

The Cochrane Library: CD ROM Update Software Ltd, Oxford, 2003. http://www.cochrane. co.uk.

World Health Organization: A Guide to Oral Health Epidemiological Investigations. Geneva, World Health Organization, 1979. 